\title{
Finite difference patch dynamics for advection homogenization problems
}

\author{
Giovanni Samaey ${ }^{1}$, Dirk Roose ${ }^{1}$, and Ioannis G. Kevrekidis ${ }^{2}$ \\ 1 Department of Computer Science, K.U. Leuven, Celestijnenlaan 200A, 3000 \\ Leuven, Belgium. \{giovanni.samaey, dirk.roose\}@cs.kuleuven.ac.be \\ 2 Department of Chemical Engineering and PACM, Princeton University, \\ Princeton, USA. yannis.kevrekidis@princeton.edu
}

Summary. We consider problems in which there is a separation between the (microscopic) scale at which the available model is defined, and the (macroscopic) scale of interest. For time-dependent multi-scale problems of this type, an "equation-free" framework has been proposed, of which patch dynamics is an essential component. Patch dynamics is designed to perform numerical simulations of an unavailable macroscopic equation on macroscopic time and length scales; it only uses appropriately initialized simulations of the available microscopic model in a number of small boxes (patches), which cover a fraction of the space-time domain. We review some recent convergence results and demonstrate that the method allows to simulate advection-dominated problems accurately.

\section{Introduction}

In many problems of current interest, one is interested in the behaviour of a (physical, chemical) system on macroscopic length and time scales, while the only valid model is available at a more microscopic scale. For example, in polymer flow, it is often impossible to find a closed formula for the stress tensor in terms of the velocity field. Therefore, the macroscopic model (a partial differential equation, PDE) needs to be supplemented with a Monte Carlo simulation to estimate the stress tensor [19, 20, 28]. Similar problems arise in flow through porous media, where it is often hard to obtain an effective permeability coefficient analytically [2], or bacterial chemotaxis, where a PDE for the density can only be derived from an individual-based model under simplifying assumptions which cannot always be fully justified [10].

In this work, we consider situations where only a microscopic model is known,

$$
\partial_{t} u(\mathbf{x}, t)=f(u(\mathbf{x}, t)),
$$

in which $u(\mathbf{x}, t)$ represents the microscopic state variables, $\mathbf{x} \in D_{m}$ are the remaining microscopic independent variables, and $\partial_{t}$ denotes the time deriva- 
tive. We assume that an equivalent macroscopic model exists, but cannot be obtained in closed form. We denote this model by

$$
\partial_{t} U(\mathbf{X}, t)=F(U(\mathbf{X}, t)),
$$

in which $U(\mathbf{X}, t)$ represents the macroscopic state variables, and $\mathbf{X} \in D_{M}$ and $t$ are the macroscopic independent variables. If one is only interested in the macroscopic solution $U(\mathbf{X}, t)$, one can construct a so-called coarse-grained time-stepper as proposed by Kevrekidis et al [21, 36]. We introduce a timestepper for the microscopic evolution law (1),

$$
u(\mathbf{x}, t+\mathrm{d} t)=s(u(\mathbf{x}, t) ; \mathrm{d} t),
$$

where $\mathrm{d} t$ is the size of the (microscopic) time-step, and the aim is to obtain a coarse-grained time-stepper for the variables $U(\mathbf{X}, t)$ as

$$
U(\mathbf{X}, t+\delta t)=\bar{S}(U(\mathbf{X}, t) ; \delta t),
$$

where $\delta t$ denotes the size of the (coarse-grained) time-step, and the bar has been introduced to emphasize the fact that the time-stepper for the macroscopic variables is only an approximation of a time-stepper for (2), since this equation is not explicitly known.

To define a coarse-grained time-stepper (4), we need to introduce two operators that make the transition between microscopic and macroscopic variables. We define a lifting operator,

$$
\mu: U(\mathbf{X}, t) \mapsto u(\mathbf{x}, t)=\mu(U(\mathbf{X}, t)),
$$

which maps macroscopic to microscopic variables, and its complement, the restriction operator

$$
\mathcal{M}: u(\mathbf{x}, t) \mapsto U(\mathbf{X}, t)=\mathcal{M}(u(\mathbf{x}, t)) .
$$

The restriction operator can often be determined as soon as the macroscopic variables are known. For instance, when the microscopic model consists of an evolving ensemble of many particles, the restriction typically consists of the computation of the low-order moments of the distribution (density, momentum, energy), which are considered as the macroscopic variables $U(\mathbf{X}, t)$. The assumption that a macroscopic equation exists for these low-order moments, implies that the higher-order moments become functionals of the low-order moments on time-scales which are fast compared to the overall system evolution (slaving).

The construction of the lifting operator is usually more involved. Again taking the example of a particle model, we need to define a mapping from a few low-order moments to an initial condition for each of the particles. We know that the higher-order moments of the resulting particle distribution should be functionals of the low-order moments, but unfortunately, these functionals are unknown (since the macroscopic evolution law is also unknown). 
Several approaches have been suggested to address this problem. One could for instance initialize the higher-order moments randomly. This introduces a lifting error, and one then relies on the separation of time-scales to ensure that these higher-order moments relax quickly to a functional of the low-order moments (healing) [13, 25, 35]. We note that, in some cases, this approach can be shown to produce inaccurate results [22]. In fact, to initialize the higherorder moments correctly, one should perform a simulation of the microscopic system, with the additional constraint that the low-order moments should be kept fixed. How this can be done using only a time-stepper for the original microscopic system, is explained and analyzed in [11, 12, 23].

Given an initial condition for the macroscopic variables $U\left(\mathbf{X}, t^{*}\right)$ at some time $t^{*}$, we can then construct the time-stepper (4) in the following way:

1. Lifting. Using the lifting operator (5), create appropriate initial conditions $u\left(\mathbf{x}, t^{*}\right)$ for the microscopic time-stepper (3), consistent with the macroscopic variables.

2. Simulation. Use the time-stepper (3) to compute the microscopic state $u(\mathbf{x}, t)$ for $t \in\left[t^{*}, t^{*}+\delta t\right]$.

3. Restriction. Obtain the macroscopic state $U\left(\mathbf{X}, t^{*}+\delta t\right)$ from the microscopic state $u\left(\mathbf{x}, t^{*}+\delta t\right)$ using the restriction operator $(6)$.

Assuming $\delta t=k \mathrm{~d} t$, this can be written as

$$
U(\mathbf{X}, t+\delta t)=\bar{S}(U(\mathbf{X}, t), \delta t)=\mathcal{M}\left(s^{k}(\mu(U(\mathbf{X}, t)), \mathrm{d} t)\right),
$$

where we have represented the $k$ microscopic time-steps by a superscript on $s$. If the microscopic model is stochastic, one may need to perform multiple replica simulations to get an accurate result.

Here, we consider situations where the macroscopic model (2) is assumed to be a partial differential equation in one space dimension, so $\mathbf{X}=x$. For this type of problems, the patch dynamics scheme was proposed [21,32,33], which only performs appropriately initialized microscopic simulations in a small fraction of the space-time domain to reduce the computational cost. The general idea is the following. First, we construct a coarse time-stepper which only performs simulations of the microscopic model in a number of small boxes, which can be thought of as macroscopic mesh points. We initialize a microscopic simulation at time $t^{*}$ in each of the boxes (lifting); run the time-stepper (3) until $t=t^{*}+\delta t$ and compute the macroscopic variables in each of the boxes at time $t^{*}+\delta t$ (restriction). The resulting coarse-grained time-stepper is called the gap-tooth scheme [21,33]. Because the microscopic time-stepper (3) takes very small time-steps of size $\mathrm{d} t$, the coarse-grained time-step $\delta t$ may still be very small compared to the slow time-scales of the macroscopic model (2). Therefore, we use the gap-tooth time-stepper to estimate the macroscopic time derivative and use this estimate to take a time-step of size $\Delta t \gg \delta t$.

The performance and accuracy of the patch dynamics scheme are currently under active investigation. Recently, we have studied the convergence properties patch dynamics scheme for a model diffusion homogenization problem. 
We showed that the patch dynamics scheme approximates a finite difference scheme for the effective (homogenized) equation, using only the microscopic (homogenization) equation in a set of small boxes [31, 32, 33]. A major issue is the imposition of appropriate box boundary conditions. For example, when the macroscopic behaviour is governed by diffusion, we can impose the average gradient as a boundary condition [33], or we can take arbitrary boundary conditions, provided we surround the computational boxes by buffer boxes to reduce the artefacts [32]. This latter technique is especially suited when a (e.g. particle) code is given, with built-in boundary conditions which are impossible, or very difficult, to change. Roberts et al. are investigating boundary conditions that lead to higher order accurate schemes [29].

In this paper, we confine ourselves to homogenization problems for the purpose of convergence analysis. In this case, the microscopic model is a partial differential equation with coefficients that vary on a small spatial scale, while the macroscopic model is a partial differential equation for the effective behaviour on large spatial scales. However, we emphasize that the method can also be applied with, and is in fact designed for, the effective behaviour of truly microscopic models, such as kinetic Monte Carlo methods, or molecular dynamics.

We note that many numerical schemes have been devised for the homogenization problem. The earliest work dates back to Babuska [3] for elliptic problems and Engquist [8] for dynamic problems. Without the aim of being complete, we mention some recent multi-scale approaches to the homogenization problem. The multi-scale finite element method of Hou and Wu uses special basis functions to capture the correct microscopic behaviour [16, 17]. Schwab, Matache and Babuska have devised a generalized FEM method based on a two-scale finite element space [26,34]. Other approaches include the use of wavelet projections [6,9] and multi-grid cycles [27]. Runborg et al. [30] proposed a time-stepper based method that obtains the effective behaviour through short bursts of detailed simulations appropriately averaged over many shifted initial conditions. The simulations were performed over the whole domain, but the notion of effective behaviour is identical. In their recent work, $\mathrm{E}$ and Engquist and collaborators address the same problem of simulating only the macroscopic behaviour of a multiscale model, see e.g. $[1,7]$. In their method, which is very similar in spirit, an unavailable macroscopic flux is estimated from appropriately initialized and constrained microscopic simulations, and used inside a macroscopic finite volume scheme.

The paper is organized as follows. In section 2 we discuss some model homogenization problems. Section 3 explains the patch dynamics scheme. We briefly review some theoretical convergence results in section 4 . In section 5 , we show that we can also approximate the macroscopic behaviour of hyperbolic homogenization problems. This is possible because we can approximate any desired finite difference scheme by an appropriate choice of the lifting step (the initialization of the small boxes). We note that the theoretical convergence analysis has not explicitly been done for this case. We conclude in section 6 . 


\section{Model problems}

\subsection{Parabolic homogenization problem}

As a microscopic problem, we consider a parabolic partial differential equation,

$$
\begin{aligned}
\partial_{t} u_{\epsilon}(x, t) & =\partial_{x}\left(a(x / \epsilon) \partial_{x} u_{\epsilon}(x, t)\right), \\
u_{\epsilon}(x, 0) & =u^{0}(x) \in L^{2}([0,1]) \\
u_{\epsilon}(0, t) & =u_{\epsilon}(1, t)=0
\end{aligned}
$$

where $a(y)=a(x / \epsilon)$ is uniformly elliptic and periodic in $y$ and $\epsilon$ is a small parameter. We choose homogeneous Dirichlet boundary conditions for simplicity.

On the macroscopic scale, we are interested in an effective, homogenized partial differential equation, in which the small-scale parameter $\epsilon$ has been eliminated. According to classical homogenization theory [4], the solution of (8) can be written as an asymptotic expansion in $\epsilon$,

$$
u_{\epsilon}(x, t)=U(x, t)+\sum_{i=1}^{\infty} \epsilon^{i}\left(u_{i}(x, x / \epsilon, t)\right) \text {, }
$$

where the functions $u_{i}(x, y, t) \equiv u_{i}(x, x / \epsilon, t), i=1,2, \ldots$ are periodic in $y$. Here, $U(x, t)$ is the solution of the homogenized equation

$$
\begin{aligned}
\partial_{t} U(x, t) & =\partial_{x}\left(a^{*} \partial_{x} U(x, t)\right) \\
U(x, 0) & =u^{0}(x) \in L^{2}([0,1]), \\
U(0, t) & =U(1, t)=0 .
\end{aligned}
$$

Here, $a^{*}$ is the constant effective coefficient, given by

$$
a^{*}=\int_{0}^{1} a(y)\left(1-\frac{\mathrm{d}}{\mathrm{d} y} \chi(y)\right) \mathrm{d} y
$$

and $\chi(y)$ is the periodic solution of

$$
\frac{\mathrm{d}}{\mathrm{d} y}\left(a(y) \frac{\mathrm{d}}{\mathrm{d} y} \chi(y)\right)=\frac{\mathrm{d}}{\mathrm{d} y} a(y),
$$

the so-called cell problem. The solution of (12) is only defined up to an additive constant, so we impose the extra condition

$$
\int_{0}^{1} \chi(y) \mathrm{d} y=0 .
$$

We note that in one space dimension, an explicit formula is known for $a^{*}$ [4], 


$$
a^{*}=\left[\int_{0}^{1} \frac{1}{a(y)} \mathrm{d} y\right]^{-1} .
$$

These asymptotic expansions have been rigorously justified in the classical book [4], see also [5]. Under the smoothness assumptions made on $a(x / \epsilon)$, one obtains strong convergence of $u_{\epsilon}(x, t)$ to $U(x, t)$ as $\epsilon \rightarrow 0$ in $L^{2}([0,1]) \times$ $C([0, T))$. Indeed, we can write

$$
\left\|u_{\epsilon}(x, t)-U(x, t)\right\|_{L_{2}([0,1])} \leq C_{0} \epsilon,
$$

uniformly in $t$.

\subsection{Hyperbolic homogenization problem}

We consider the following hyperbolic partial differential equation in one space dimension,

$$
\begin{aligned}
\partial_{t} u_{\epsilon}(x, t) & +\partial_{x}\left[c(x / \epsilon) u_{\epsilon}(x, t)\right]=0, \\
u_{\epsilon}(x, 0) & =u^{0}(x) \in L^{2}([0,1]), \quad \partial_{x} u_{\epsilon}(0, t)=0,
\end{aligned}
$$

where $c(y)=c(x / \epsilon)>0$ is periodic in $y$ and $\epsilon$ is a small parameter. We choose a homogeneous Neumann boundary condition for simplicity.

As in the previous section, we are interested in an effective, homogenized partial differential equation on a macroscopic scale, where the dependence on the small scale parameter has been eliminated. According to classical homogenization theory $[4,5]$, the solution of (15) converges weakly in the limit of $\epsilon \rightarrow 0$ to the solution of

$$
\begin{aligned}
\partial_{t} U(x, t) & +\partial_{x}\left[c^{*} U(x, t)\right]=0, \\
U(x, 0) & =u^{0}(x) \in L^{2}([0,1]), \quad \partial_{x} U(0, t)=0,
\end{aligned}
$$

which describes the evolution of the averaged, effective behaviour. As in the parabolic case, the effective coefficient $c^{*}$ is given by the harmonic average,

$$
c^{*}=\left[\int_{0}^{1} \frac{1}{c(y)} \mathrm{d} y\right]^{-1} .
$$

\section{Patch dynamics}

We devise a scheme for the evolution of the effective behaviour $U(x, t)$ of a general homogenization problem,

$$
\partial_{t} u_{\epsilon}=f\left(u_{\epsilon}, \partial_{x} u_{\epsilon}, \ldots, \partial_{x}^{d} u_{\epsilon}, t ; \epsilon\right) .
$$

We assume that a time integration code for this equation has already been written and is available with a number of standard boundary conditions, such 
as no-flux or Dirichlet. Further, we assume that the macroscopic equation is of the form

$$
\partial_{t} U=F\left(U, \partial_{x} U, \ldots, \partial_{x}^{d} U, t\right),
$$

in which the order of the equation (the highest spatial derivative) $d$ is assumed to be known. For a strategy to obtain such information, see [24].

Suppose we want to obtain the solution of (19) on the interval $[0,1]$, using an equidistant, macroscopic mesh $\Pi(\Delta x):=\left\{0=x_{0}<x_{1}=x_{0}+\Delta x<\right.$ $\left.\ldots<x_{N}=1\right\}$. Given equation (19), we can define a method-of-lines space discretization,

$$
\partial_{t} U_{i}(t)=F\left(U_{i}(t), D^{1}\left(U_{i}(t)\right), \ldots, D^{d}\left(U_{i}(t)\right), t\right), \quad i=0, \ldots, N .
$$

where $U_{i}(t) \approx U\left(x_{i}, t\right)$ and $D^{k}\left(U_{i}(t)\right)$ denotes a suitable finite difference approximation for the $k$-th spatial derivative. We subsequently discretize equation (20) in time using a time integration method of choice, e.g. forward Euler. We denote the resulting time-stepper as

$$
U^{n+1}=S\left(U^{n}, t_{n} ; \Delta t\right)=U^{n}+\Delta t F\left(U^{n}, t_{n}\right),
$$

where $U^{n}=\left(U_{0}\left(t_{n}\right), \ldots, U_{N}\left(t_{n}\right)\right)^{T}$ and $\Delta t$ denotes the macroscopic time-step. We have suppressed the dependence of $F\left(U^{n}, t_{n}\right)$ on the spatial derivatives for notational convenience. Note that, although we have used the forward Euler scheme here for concreteness, in principle any time discretization method can be used to solve equation (20).

Since equation (19) is assumed not to be known explicitly, we will use (21) for analysis purposes only. We construct a (patch dynamics) scheme to approximate (21). To this end, we consider a small interval (box, tooth) of size $h \ll \Delta x$ around each mesh point, and define the discrete solution $\bar{U}(t)=\left(\bar{U}_{0}(t), \ldots, \bar{U}_{N}(t)\right)^{T} \in \mathbb{R}^{N+1}$ as being the average of the microscopic solution in the small boxes,

$$
\bar{U}_{i}(t)=\mathcal{S}_{h}\left(u_{\epsilon}\right)\left(x_{i}, t\right)=(1 / h) \int_{x_{i}-h / 2}^{x_{i}+h / 2} u_{\epsilon}(\xi, t) d \xi, \quad i=0, \ldots, N .
$$

We denote an approximation of $\bar{U}(t)$ at $t=t_{n}$ as $\bar{U}^{n}$.

The patch dynamics scheme is now constructed as follows. We introduce a larger buffer box of size $H>h$ around each mesh point (see figure 1.) In each box of size $H$, we perform a time integration over a time interval of size $\delta t$ using the microscopic model (18), and restrict to macroscopic variables. The results are used to estimate the macroscopic time derivative. We provide each microscopic simulation with the following initial and boundary conditions.

Initial condition. We define the initial condition by constructing a local Taylor expansion, based on the (given) box averages $\bar{U}_{i}^{n}, i=0, \ldots, N$, at mesh point $x_{i}$ and time $t_{n}$

$$
\bar{u}_{\epsilon}^{i}\left(x, t_{n}\right)=\sum_{k=0}^{d} D_{i}^{k}\left(\bar{U}^{n}\right) \frac{\left(x-x_{i}\right)^{k}}{k !}, \quad x \in\left[x_{i}-\frac{H}{2}, x_{i}+\frac{H}{2}\right],
$$




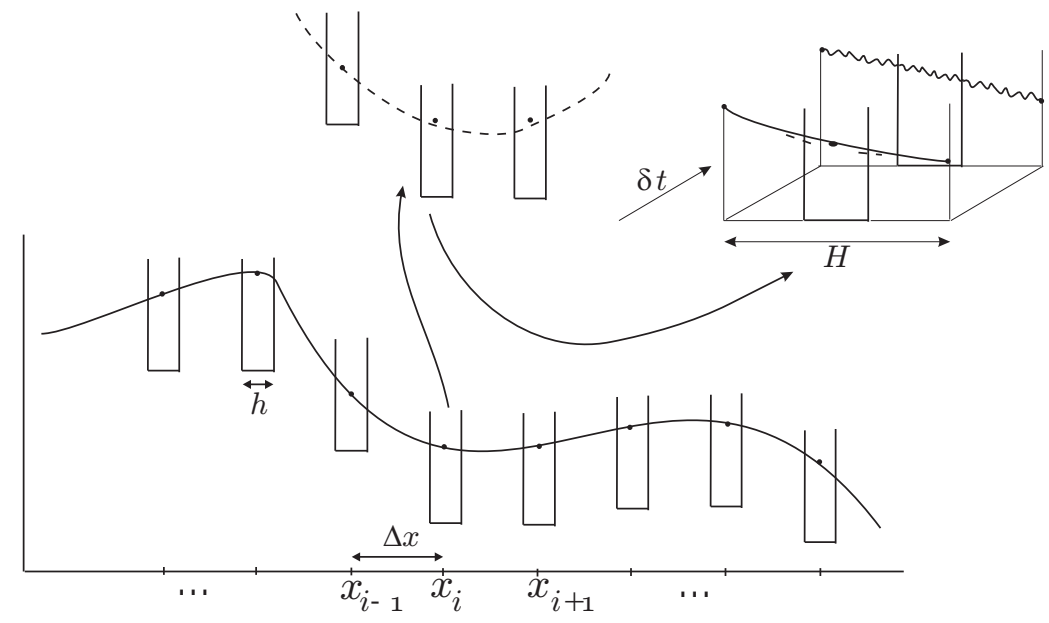

Fig. 1: A schematic representation of the gap-tooth scheme with buffer boxes. We choose a number of boxes of size $h$ around each macroscopic mesh point $x_{i}$ and define a local Taylor approximation as initial condition in each box. Simulation is performed inside the larger (buffer) boxes of size $H$, where some boundary conditions are imposed.

where $d$ is the order of the macroscopic equation (19). The coefficients $D_{i}^{k}\left(\bar{U}^{n}\right)$, $k>0$ are the same finite difference approximations for the $k$-th spatial derivative that would be used in the comparison scheme $(20)$, whereas $D_{i}^{0}\left(\bar{U}^{n}\right)$ is chosen such that

$$
\frac{1}{h} \int_{x_{i}-h / 2}^{x_{i}+h / 2} \bar{u}_{\epsilon}^{i}\left(\xi, t_{n}\right) \mathrm{d} \xi=\bar{U}_{i}^{n} .
$$

Boundary conditions. The time integration of the microscopic model in each box should provide information on the evolution of the global problem at that location in space. It is therefore crucial that the boundary conditions are chosen such that the solution inside each box evolves as if it were embedded in the larger domain. To this end, we introduce a larger box of size $H>h$ around each macroscopic mesh point. The simulation can subsequently be performed using any of the built-in boundary conditions of the microscopic code. Lifting and (short-term) evolution (using arbitrary available boundary conditions) are performed in the larger box; yet the restriction is done by processing the solution (here taking its average) over the inner, small box only. The goal of the additional computational domains, the buffers, is to buffer the solution inside the small box from the artificial disturbance caused by the (repeatedly updated) boundary conditions. This can be accomplished over short enough time intervals, provided the buffers are large enough; analyzing the method is tantamount to making these statements quantitative. 
The algorithm. The complete algorithm to obtain an estimate of the macroscopic time derivative at time $t_{n}$ is given below:

1. Lifting. At time $t_{n}$, construct the initial condition $\bar{u}_{\epsilon}^{i}\left(x, t_{n}\right), i=0, \ldots, N$ using the box averages $\bar{U}_{i}^{n}$, as defined in (23).

2. Simulation. Compute the box solution $\bar{u}_{\epsilon}^{i}(x, t), t>t_{n}$, by solving equation (18) in the interval $\left[x_{i}-H / 2, x_{i}+H / 2\right]$ with some boundary conditions up to time $t_{n+\delta}=t_{n}+\delta t$. The boundary conditions can be anything that the microscopic code allows.

3. Restriction. Compute the average $\bar{U}_{i}^{n+\delta}=1 / h \int_{x_{i}-h / 2}^{x_{i}+h / 2} \bar{u}_{\epsilon}^{i}\left(\xi, t_{n+\delta}\right) \mathrm{d} \xi$ over the inner, small box only.

4. Estimation. We estimate the time derivative at time $t_{n}$ as

$$
\bar{F}^{d}\left(\bar{U}^{n}, t_{n} ; h, \delta t, H\right)=\frac{\bar{U}^{n+\delta}-\bar{U}^{n}}{\delta t},
$$

where we have added a superscript $d$ to denote the highest spatial derivative that has been initialized in the lifting step. We also made explicit the dependence of the estimate on $H$ and $\delta t$.

Since the first three steps constitute a gap-tooth time-step, we call the estimator (25) a gap-tooth time derivative estimator. It can be used in any ODE time integration code. For example, a forward Euler patch dynamics scheme would be

$$
\bar{U}^{n+1}=\bar{U}^{n}+\Delta t \bar{F}^{d}\left(\bar{U}^{n}, t_{n} ; h, \delta t, H\right) .
$$

For more details, including a discussion of the additional issues that need to be addressed for truly microscopic models, we refer to [32]. We emphasize that an initialization according to equation (23) has the important advantage that one can choose a suitable finite difference approximation for each derivative independently, as opposed to the method described in [21,33], which automatically leads to central finite differences. This property is crucial, and will allow us to approximate advection-dominated equations more effectively.

\section{Convergence results}

In this section, we briefly review some theoretical convergence results that were obtained for the parabolic homogenization problem (8), see [32] for details. In this case, we know that the order of the macroscopic equation $d=2$.

\subsection{Consistency analysis}

For the effective equation (10), one can write a finite-difference/forward Euler time-stepper as follows, 


$$
\begin{aligned}
U^{n+\delta} & =S\left(U^{n}, t_{n} ; \delta t\right) \\
& =U^{n}+\delta t F\left(U^{n}, D^{1}\left(U^{n}\right), D^{2}\left(U^{n}\right), t_{n}\right) \\
& =U^{n}+\delta t\left[a^{*} D^{2}\left(U^{n}\right)\right] .
\end{aligned}
$$

We compare the gap-tooth time-derivative estimator with the effective time derivative. For concreteness, we impose Dirichlet boundary conditions at the boundaries of the boxes, which will clearly introduce artefacts on the estimated time derivative. The subsequent theorem shows that these artefacts can be made arbitrarily small by increasing the buffer size $H$ [32].

Theorem 1 (Consistency) Let $\bar{F}^{2}\left(\bar{U}^{n}, t_{n} ; h, \delta_{t}, H\right)$ be a gap-tooth timestepper for the homogenization problem (8). Then, assuming $U^{n}=\bar{U}^{n}$, we have,

$$
\begin{aligned}
& \| \bar{F}^{2}\left(\bar{U}^{n}, t_{n} ; \delta t, H\right)-a^{*} D^{2}\left(U^{n}\right) \| \leq \\
& C_{4} \underbrace{}_{\text {micro-scales }} \underbrace{\frac{\epsilon}{\sqrt{h} \delta t}}_{\text {averaging }}+C_{5} \underbrace{\left(1+\frac{h^{2}}{\delta t}\right)}_{\text {boundary conditions }}
\end{aligned}
$$

Formula (28) shows the main consistency properties of the gap-tooth estimator. The error decays exponentially as a function of buffer size, but the optimal accuracy of the estimator is limited by the presence of the microscopic scales. Therefore, we need to make a trade-off to determine an optimal choice for $H$ and $\delta t$. The smaller $\delta t$, the smaller $H$ can be used to reach optimal accuracy (and thus the smaller the computational cost), but smaller $\delta t$ implies a larger optimal error.

It is shown numerically in [32] that the convergence result does not depend crucially on the type of boundary conditions. E.g. for no-flux boundary conditions, we obtain qualitatively the same result. However, if we know how the macroscopic solution behaves at the boundaries of the boxes, we can use this knowledge to eliminate the buffers. For the diffusion problem, we have shown that we do not need buffer regions if we constrain the macroscopic gradient at the boundaries [33]. However, in general it is very difficult to find and implement such constraints for a given microscopic simulator.

\subsection{Stability}

Theorem 1 establishes the consistency of the gap-tooth scheme. To obtain convergence, we also need stability. In [7], E and Engquist state that the heterogeneous multiscale method is stable if the corresponding comparison scheme is stable, see [7, Theorem 5.5]. This theorem would also apply to our case. However, due to the a priori assumption that the numerical approximation remains bounded, it may be of little practical value. Here, we circumvent some 
of these difficulties by studying the stability properties of the scheme numerically. This can be done by computing the eigenvalues of the time derivative estimator as a function of $H$.

Consider the homogenization diffusion equation (8) with the diffusion coefficient $a(x / \epsilon)=1.1+\sin (2 \pi x / \epsilon)$. The homogenized equation is given by (10) with $a^{*}=0.45825686$.

We define the concrete patch dynamics scheme to be a forward Euler scheme,

$$
\bar{U}^{n+1}=U^{n}+\Delta t \bar{F}^{2}\left(\bar{U}^{n}, t_{n} ; \delta t, H\right),
$$

with the box initialization defined by (23) with second order central finite differences. In this case, the comparison finite difference scheme for the macroscopic equation is given by

$$
U^{n+1}=U^{n}+\Delta t F\left(U^{n}, t_{n}\right)=U^{n}+a^{*} \Delta t \frac{U_{i+1}^{n}-2 U_{i}^{n}+U_{i-1}^{n}}{\Delta x^{2}}
$$

The time derivative operator $F\left(U^{n}, t_{n}\right)$ in the comparison scheme (30) has eigenvalues

$$
\lambda_{k}=-\frac{4 a^{*}}{\Delta x^{2}} \sin ^{2}(\pi k \Delta x),
$$

which, using the forward Euler scheme as time-stepper, results in the stability condition

$$
\max _{k}\left|1+\lambda_{k} \Delta t\right| \leq 1 \quad \text { or } \quad \frac{\Delta t}{\Delta x^{2}} \leq \frac{1}{2} a^{*}
$$

It can easily be checked that the operator $\bar{F}^{2}\left(U^{n}, t_{n} ; \delta t, H\right)$ is linear, so we can interpret the evaluation of $\bar{F}^{2}\left(U^{n}, t_{n} ; \delta t, H\right)$ as a matrix-vector product. We can therefore use any matrix-free linear algebra technique to compute the eigenvalues of $\bar{F}^{2}\left(U^{n}, t_{n} ; \delta t, H\right)$, e.g. Arnoldi [14]. We choose to compute $\bar{F}^{2}\left(U^{n}, t_{n} ; \delta t, H\right)$ and $F\left(U^{n}, t_{n}\right)$ on the domain $[0,1]$ with Dirichlet boundary conditions, on a mesh of width $\Delta x=0.05$ and with an inner box width of $h=2 \cdot 10^{-3}$. We choose $\delta t=5 \cdot 10^{-6}$ and compute the eigenvalues of $\bar{F}^{2}\left(U^{n}, t_{n} ; \delta t, H\right)$ as a function of $H$. The results are shown in figure 2 . When the buffer size is too small, the eigenvalues of the gap-tooth estimator are closer to 0 than the corresponding eigenvalues of the finite difference scheme. This is because the microscopic simulation approaches a steady state quickly (due to the Dirichlet boundary conditions), instead of following the true system evolution in a larger domain. With increasing buffer size $H$, the eigenvalues of $\bar{F}^{2}\left(U^{n}, t_{n} ; \delta t, H\right)$ approximate those of $F\left(U^{n}, t_{n}\right)$, which is an indication of consistency for larger $H$. Since all eigenvalues are negative and the most negative eigenvalue for $\bar{F}^{2}\left(U^{n}, t_{n} ; \delta t, H\right)$ is always smaller in absolute value than the corresponding eigenvalue of $F\left(U^{n}, t_{n}\right)$, the patch dynamics scheme is always stable if the comparison scheme is stable. 


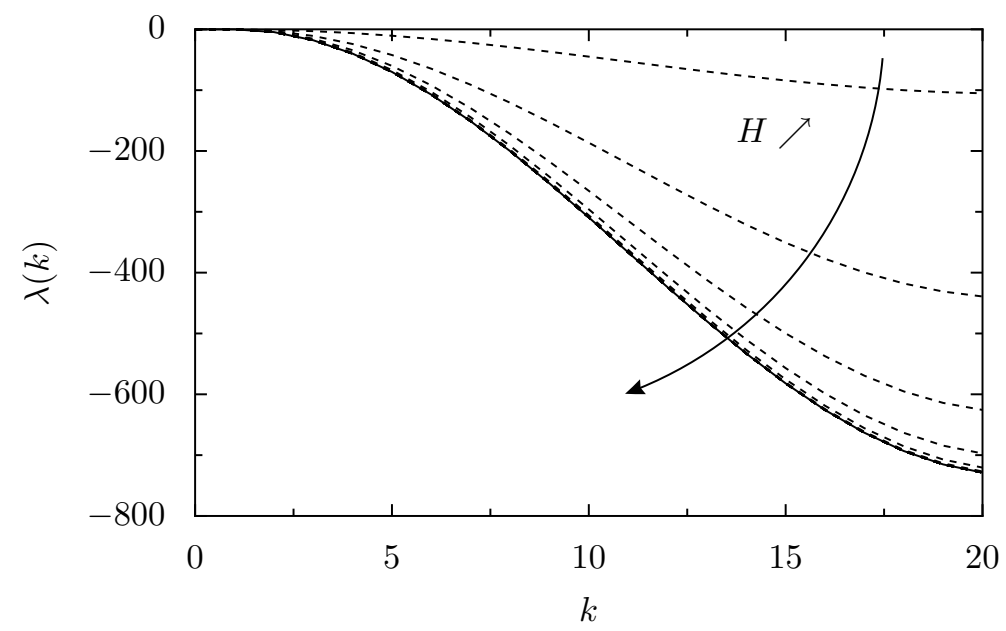

Fig. 2: Spectrum of the estimator $\bar{F}^{2}\left(U^{n}, t_{n} ; \delta t, H\right)$ (dashed) for the model equation (8) for $H=2 \cdot 10^{-3}, 4 \cdot 10^{-3}, \ldots, 2 \cdot 10^{-2}$ and $\delta t=5 \cdot 10^{-6}$, and the eigenvalues (31) of $F\left(U^{n}, t_{n}\right)$ (solid).

\subsection{Numerical illustration}

We illustrate the theory with a diffusion homogenization problem. Consider the model problem (8) with

$$
a(x / \epsilon)=1.1+\sin (2 \pi x / \epsilon), \quad \epsilon=1 \cdot 10^{-5}
$$

as a microscopic problem on the domain $[0,1]$ with homogeneous Dirichlet boundary conditions and initial condition $u(x, 0)=1-4(x-1 / 2)^{2}$. The corresponding macroscopic equation is given by equation (10), with $a^{*}=$ 0.45825686 . This problem has also been used as a model example in $[1,33]$. To solve this microscopic problem, we use a second order finite difference discretization with mesh width $\delta x=1 \cdot 10^{-7}$ and lsode [15] as time-stepper. The concrete gap-tooth scheme for this example is again defined by taking second order central finite differences.

We first perform a numerical experiment to show the convergence behaviour in terms of buffer width. Once a suitable buffer width has been determined, we perform a long term simulation.

Buffer width. We first compare a gap-tooth step with $h=2 \cdot 10^{-3}$ and $\Delta x=1 \cdot 10^{-1}$ with the reference estimator $a^{*} D^{2}\left(\hat{U}^{n}\right)$, in which the effective diffusion coefficient is known to be $a^{*}=0.45825686$. Figure 3 shows the error with respect to the finite difference time derivative as a function of $H$ (left) and $\delta t$ (right). It is clear that the convergence is in agreement with Theorem 1 . We see that smaller values of $\delta t$ result in larger values for the optimal error, but the convergence towards this optimal error is faster. 

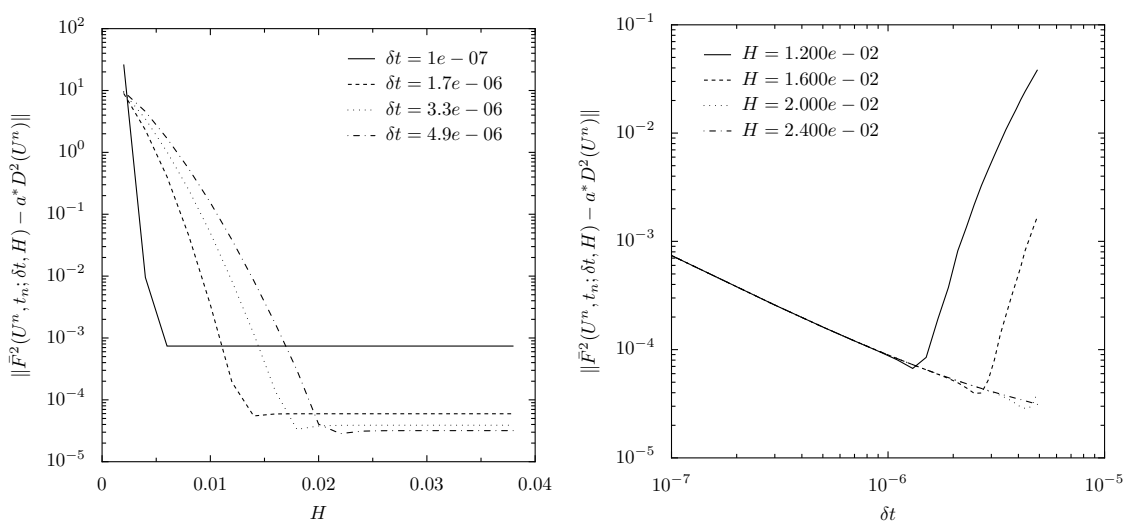

Fig. 3: Error of the gap-tooth estimator $\bar{F}^{2}\left(U^{n}, t_{n} ; \delta t, H\right)$ with respect to the finite difference time derivative $a^{*} D^{2}\left(U^{n}\right)$ on the same mesh. Left: Error with respect to $H$ for fixed $\delta t$. Right: Error with respect to $\delta t$ with fixed $H$.

Long term simulation. We now perform a long term simulation and compare the results with a long term simulation using the comparison scheme. The properties for the macroscopic scheme are chosen to be $\Delta x=1 \cdot 10^{-1}$ and $\Delta t=1 \cdot 10^{-3}$. As gap-tooth parameters, we choose $H=8 \cdot 10^{-3}, \delta t=1 \cdot 10^{-6}$ and $h=1 \cdot 10^{-4}$. Thus, simulations are performed in only $8 \%$ of the spatial domain, and $0.1 \%$ of the time domain. The results are shown in figure 4 . We also compare the results of the patch dynamics scheme to a reference solution of the effective equation, which is obtained using the comparison scheme on a much finer grid $\left(\Delta x=5 \cdot 10^{-3}\right.$ and $\left.\Delta t=1 \cdot 10^{-6}\right)$. We see that the solution is well approximated, and that the error of the patch dynamics scheme with respect to the finite difference comparison scheme is an order of magnitude smaller than the total error with respect to the reference solution.

\section{Numerical results for advection problems}

Consider equation (15) with

$$
c(x / \epsilon)=1 /(3+\sin (2 \pi x / \epsilon)), \quad \epsilon=1 \cdot 10^{-5} .
$$

The effective equation is then given by (16) with $c^{*}=1 / 3$. The available microscopic simulation code is an upwind/forward Euler time-stepper on a grid with size $\delta x=5 \cdot 10^{-10}$ and a time-step $\mathrm{d} t=5 \cdot 10^{-11}$. We take the size of the small boxes to be $h=5 \cdot 10^{-4}$.

We first investigate how the accuracy of the scheme is influenced by the buffer size $H$ and the gap-tooth time-step $\delta t$. Once a good set of method parameters is found, we perform a long-term simulation. We construct patch 

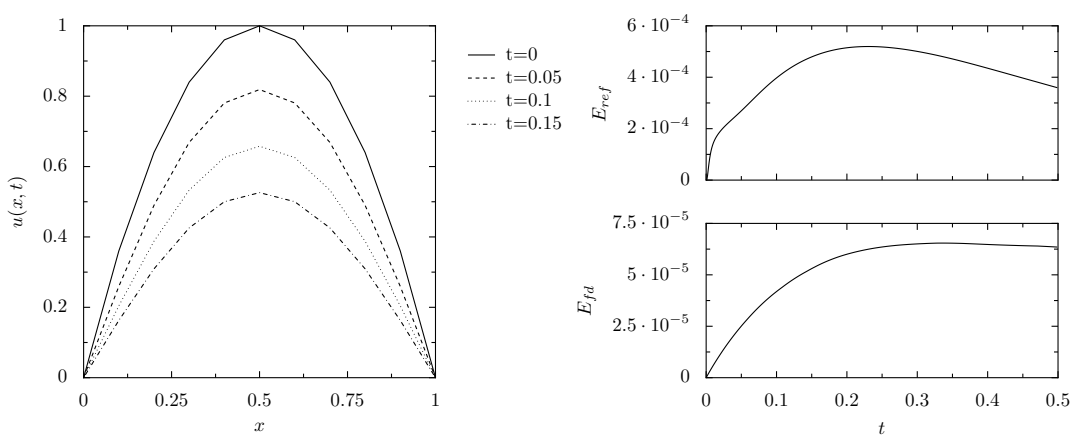

Fig. 4: Left: Snapshots of the solution of the homogenization diffusion equation using the patch dynamics scheme at certain moments in time. Right: error with respect to the "exact" solution of the effective equation (top) and a finite difference comparison scheme (bottom). The total error is dominated by the error of the finite difference scheme.

dynamics schemes to mimic the upwind, third-order upwind-biased and central fourth-order spatial discretizations.

\subsection{Consistency}

To determine the buffer size $H$ and the gap-tooth time-step $\delta t$, we perform a numerical simulation for this model on the domain $[-H / 2,+H / 2]$, with $H=h+5 i \cdot 10^{-9}$ for $i=1, \ldots, 20$ on the time interval $[0, \delta t]$ with $\delta t=j \cdot 10^{-9}$, $j=1, \ldots, 100$ and the linear initial condition

$$
u_{\epsilon}(x, 0)=D^{1} x+D^{0}=3.633 x+0.9511 .
$$

The results are shown in figure 5 (left). We notice two differences with respect to the parabolic case. First, it is clear that we do not need very large buffer regions. Indeed, the advective nature of equation (15) ensures that information travels with finite speed. The consequence is that, as soon as the time-step is too short for the boundary information to reach the interior of the domain, the buffer size $H$ will not have any influence on the accuracy of the result.

The second difference is that the error decreases monotonically with decreasing $\delta t$, whereas the theoretical result for diffusion indicates that we would have an error term of the form $O(\epsilon / \delta t)$. This discrepancy is due to additional numerical inaccuracies during the restriction step, which are caused by the weak convergence towards the homogenized equation in the hyperbolic case. Figure 5 shows how $u_{\epsilon}(x, t)$ varies as a function of time. We see that the microscopic solution develops oscillations which grow in amplitude with time. Recall that the macroscopic quantity at time $t=\delta t$ is computed as the spatial average of the solution $u_{\epsilon}(x, \delta t)$ over a box of size $h$. We need to approximate 

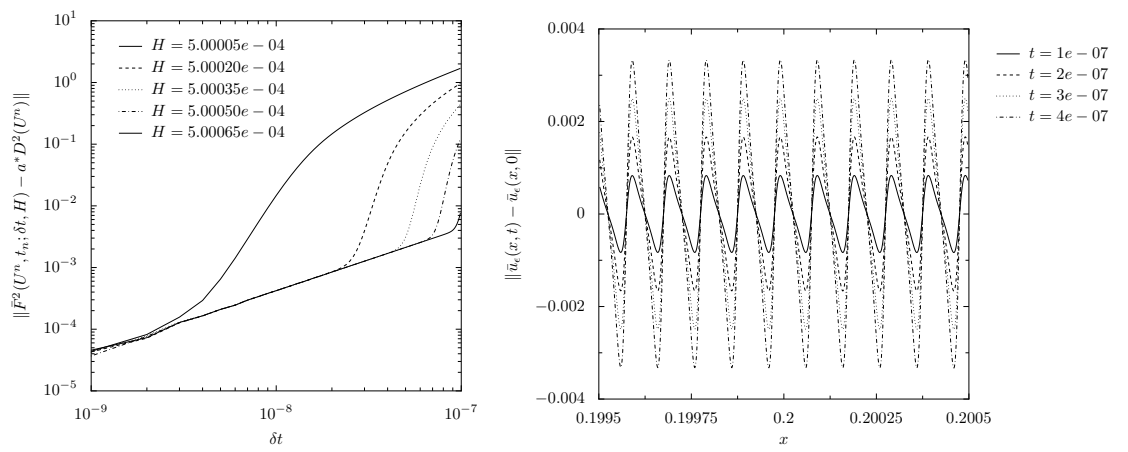

Fig. 5: Left: Error of the gap-tooth estimator with respect to the macroscopic time derivative $c^{*} D^{1}$. Right: Difference between the solution inside the box at time $t$ and the initial condition at time $t=0$.

this spatial average using a quadrature formula, in which we can only use the solution on the numerical grid points as quadrature points. Thus, we may expect a decrease of accuracy in the computation of the box average for increasing values of $\delta t$. We numerically verified this intuitive reasoning by increasing $\epsilon$. The box solution then becomes less oscillatory, and we observed that the accuracy of the restriction was increased.

Based on these results, we choose $H=h+1 \cdot 10^{-7}$ and $\delta t=5 \cdot 10^{-9}$. Since our macroscopic schemes will use $\Delta x=O\left(10^{-2}\right)$ and $\Delta t=O\left(10^{-2}\right)$, the method results in gains of the order of 100 in space and $10^{6}$ in time. However, we need to mention that, for realistic microscopic problems, part of this spectacular gain will be lost because we need to initialize the microscopic system consistently (the lifting step) using only a few low-order moments, which may require additional microscopic simulations [11, 12, 23].

\subsection{First order upwind scheme}

We perform a numerical simulation for this model on the domain $[0,1]$ with periodic boundary conditions. As an initial condition, we choose

$$
u^{0}(x)=(\sin (\pi x))^{100},
$$

which is a typical initial condition to study spatial discretizations for the advection equation [18]. We use a macroscopic mesh of size $\Delta x=1 \cdot 10^{-2}$ and a time-step $\Delta t=1 \cdot 10^{-2}$, and we define our macroscopic comparison scheme as an upwind/forward Euler scheme

$$
U_{i}^{n+1}=U_{i}^{n}-\Delta t c^{*} \frac{U_{i}^{n}-U_{i-1}^{n}}{\Delta x} .
$$


The corresponding patch dynamics scheme is defined by the algorithm in section 3, where the initial condition (23) is defined by taking $d=1$ and

$$
D_{i}^{1}\left(\bar{U}^{n}\right)=\frac{\bar{U}_{i}^{n}-\bar{U}_{i-1}^{n}}{\Delta x}, \quad D_{i}^{0}\left(\bar{U}^{n}\right)=\bar{U}_{i}^{n} .
$$

The resulting time derivative estimator is used with a forward Euler timestepper. The results are shown in figure 6 . The patch dynamics scheme clearly
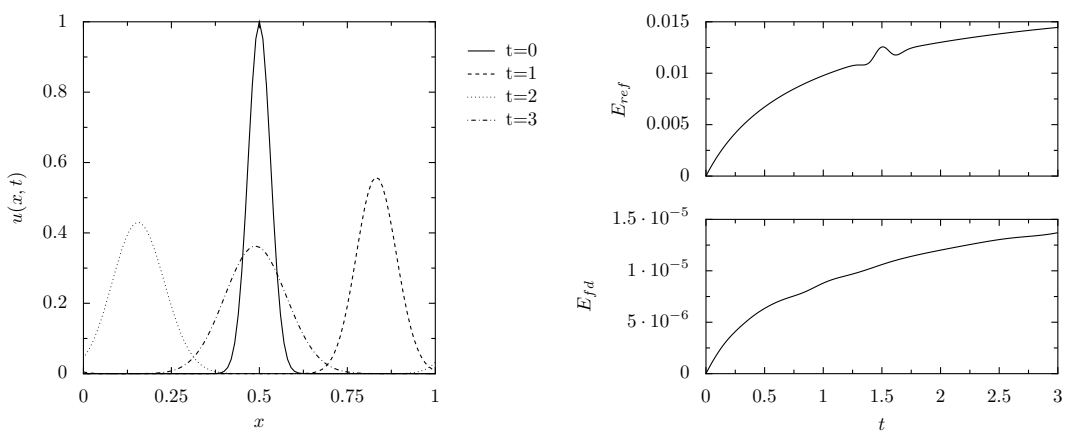

Fig. 6: Left: Snapshots of the solution of the homogenization advection equation (15) with coefficient (33) using the upwind patch dynamics scheme at certain moments in time. Right: $L_{2}$-error with respect to the "exact" solution of the effective equation (16) (top) and the finite difference comparison scheme (35) (bottom). The total error is dominated by the error of the finite difference scheme.

has the same properties as the comparison finite difference scheme. It is very diffusive, but maintains positivity. The left figure shows the $L_{2}$-error of patch dynamics with respect to the finite difference scheme, and with respect to an "exact" solution of the effective equation, which was obtained using the upwind scheme on a very fine mesh with $\Delta x=1 \cdot 10^{-4}$ and $\Delta t=1 \cdot 10^{-4}$. We see that the error of the patch dynamics scheme is completely dominated by the finite difference error.

\subsection{Third-order upwind-biased scheme}

Next, we design a patch dynamics algorithm to mimic the third-order upwindbiased scheme as a spatial discretization, which we combine with the classical fourth-order Runge-Kutta time integration method. In this case, the macroscopic time derivative is given by

$$
F\left(U_{i}^{n}, t_{n}\right)=\frac{c^{*}}{\Delta x}\left(-\frac{1}{6} U_{i-2}^{n}+U_{i-1}^{n}-\frac{1}{2} U_{i}^{n}-\frac{1}{3} U_{i+1}^{n}\right) .
$$


The Runge-Kutta method requires some auxiliary evaluations of the time derivative operator,

$$
\begin{aligned}
& k_{1}=F\left(U_{i}^{n}, t_{n}\right) \\
& k_{2}=F\left(U_{i}^{n}+\frac{\Delta t}{2} k_{1}, t_{n}+\frac{\Delta t}{2}\right) \\
& k_{3}=F\left(U_{i}^{n}+\frac{\Delta t}{2} k_{2}, t_{n}+\frac{\Delta t}{2}\right) \\
& k_{4}=F\left(U_{i}^{n}+\Delta t k_{3}, t_{n}+\Delta t\right)
\end{aligned}
$$

and the time-stepper $U^{n+1}=S\left(U^{n}, t_{n} ; \Delta_{t}\right)$ is then defined as

$$
U^{n+1}=U^{n}+\Delta t\left(\frac{1}{6} k_{1}+\frac{1}{3} k_{2}+\frac{1}{3} k_{3}+\frac{1}{6} k_{4}\right)
$$

The corresponding patch dynamics scheme is defined by the algorithm in section 3, where the initial condition (23) is defined by taking $d=1$ and

$$
\begin{aligned}
& D_{i}^{1}\left(\bar{U}^{n}\right)=\frac{1}{\Delta x}\left(\frac{1}{6} \bar{U}_{i-2}^{n}-\bar{U}_{i-1}^{n}+\frac{1}{2} \bar{U}_{i}^{n}+\frac{1}{3} \bar{U}_{i+1}^{n}\right), \\
& D_{i}^{0}\left(\bar{U}^{n}\right)=\bar{U}_{i}^{n} .
\end{aligned}
$$

The resulting time derivative estimator is subsequently used inside the fourthorder Runge-Kutta method.

We perform a numerical simulation on a macroscopic mesh with size $\Delta x=2 \cdot 10^{-2}$ and $\Delta t=2 \cdot 10^{-2}$. The results are shown in figure 7 . The patch
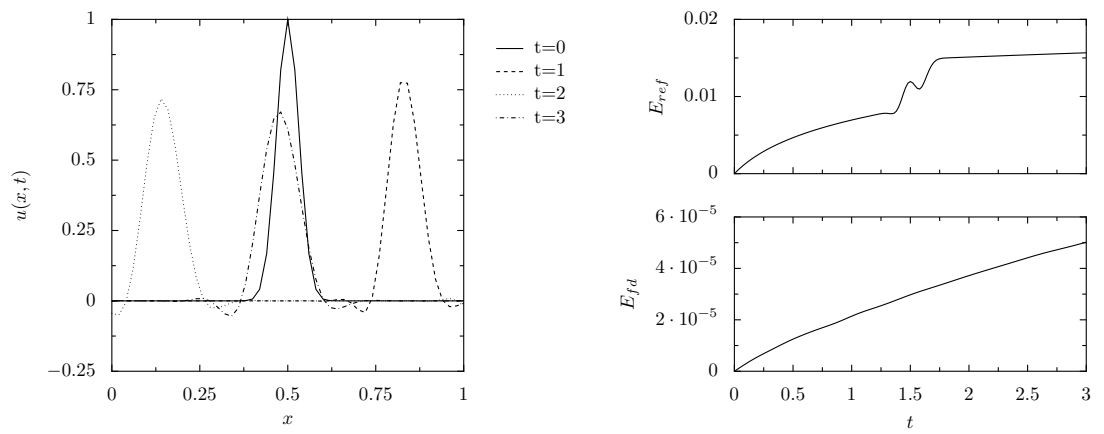

Fig. 7: Left: Snapshots of the solution of the homogenization advection equation (15) with coefficient (33) using the upwind-biased patch dynamics scheme at certain moments in time. Right: $L_{2}$-error with respect to the "exact" solution of the effective equation (16) (top) and the finite difference comparison scheme (39)-(37) (bottom). The total error is dominated by the error of the finite difference scheme.

dynamics scheme clearly has the same properties as the comparison finite 
difference scheme. It is less diffusive than the upwind scheme, but some artificial oscillations are introduced. The left figure shows the $L_{2}$-error of patch dynamics with respect to the finite difference scheme, and with respect to an "exact" solution of the effective equation, which was obtained using the upwind scheme on a very fine mesh with $\Delta x=1 \cdot 10^{-4}$ and $\Delta t=1 \cdot 10^{-4}$. Again, we see that the error in approximating the exact solution is completely dominated by the error of the macroscopic scheme, while the errors due to estimation are negligible.

\subsection{Fourth-order central scheme}

Finally, we design a patch dynamics algorithm to mimic a fourth-order central scheme as a spatial discretization, which we combine again with the classical fourth-order Runge-Kutta time integration method. In this case, the macroscopic time derivative is given by

$$
F\left(U_{i}^{n}, t_{n}\right)=\frac{c^{*}}{\Delta x}\left(-\frac{1}{12} U_{i-2}^{n}+\frac{2}{3} U_{i-1}^{n}-\frac{2}{3} U_{i+1}^{n}+\frac{1}{12} U_{i+2}^{n}\right),
$$

and the time-integration method is again given by (38)-(39). The corresponding patch dynamics scheme is defined by the algorithm in section 3 , where the initial condition (23) is defined by taking $d=1$ and

$$
\begin{aligned}
& D_{i}^{1}\left(\bar{U}^{n}\right)=\frac{1}{\Delta x}\left(\frac{1}{12} \bar{U}_{i-2}^{n}-\frac{2}{3} \bar{U}_{i-1}^{n}+\frac{2}{3} \bar{U}_{i+1}^{n}-\frac{1}{12} \bar{U}_{i+2}^{n}\right), \\
& D_{i}^{0}\left(\bar{U}^{n}\right)=\bar{U}_{i}^{n} .
\end{aligned}
$$

The resulting time derivative estimator is subsequently used inside the fourthorder Runge-Kutta method.

We perform a numerical simulation on a macroscopic mesh with size $\Delta x=$ $2 \cdot 10^{-2}$ and $\Delta t=2 \cdot 10^{-2}$. The results are shown in figure 8 . The patch dynamics scheme clearly has the same properties as the comparison finite difference scheme. It is much less diffusive than the upwind scheme, but many artificial oscillations are introduced, which is typical behaviour for central schemes. The left figure shows the $L_{2}$-error of patch dynamics with respect to the finite difference scheme, and with respect to an "exact" solution of the effective equation, which was obtained using the upwind scheme on a very fine mesh with $\Delta x=1 \cdot 10^{-4}$ and $\Delta t=1 \cdot 10^{-4}$. Again, we see that the error in approximating the exact solution is completely dominated by the error of the macroscopic scheme, while the errors due to estimation are negligible.

\subsection{Advection coefficients with macro-scale variations}

As a second example, we consider equation (15) with

$$
c(x / \epsilon)=1 /(3+\sin (2 \pi x / \epsilon)+\sin (2 \pi x)), \quad \epsilon=1 \cdot 10^{-5} .
$$



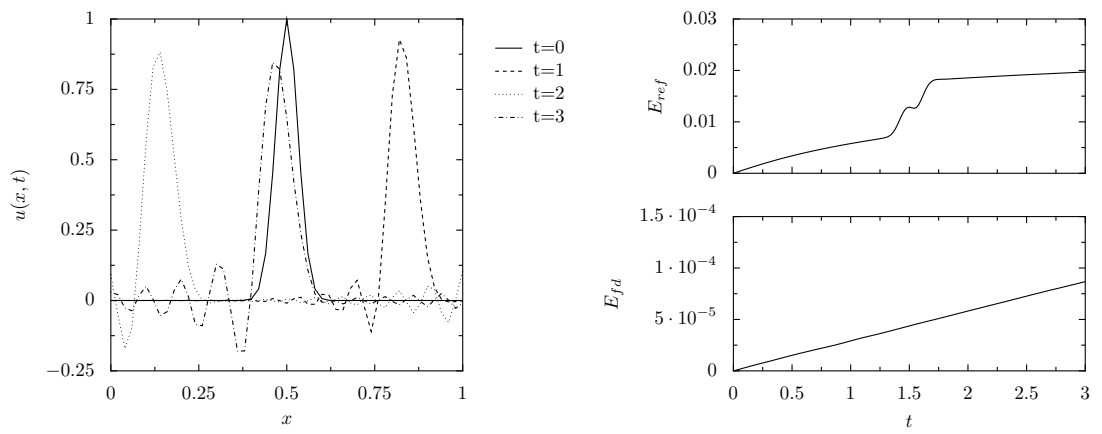

Fig. 8: Left: Snapshots of the solution of the homogenization advection equation (15) with coefficient (33) using the central fourth-order patch dynamics scheme at certain moments in time. Right: $L_{2}$-error with respect to the "exact" solution of the effective equation (16) (top) and the finite difference comparison scheme (39)-(41) (bottom). The total error is dominated by the error of the finite difference scheme.

The effective equation is then given by (16) with $c^{*}=1 /(3+\sin (2 \pi x))$. The available microscopic simulation code is an upwind/forward Euler timestepper on a grid with size $\delta x=5 \cdot 10^{-10}$ and a time-step $\mathrm{d} t=5 \cdot 10^{-11}$. We take the size of the small boxes to be $h=5 \cdot 10^{-4}$.

We choose $H=h+2 \cdot 10^{-7}$ and $\delta t=5 \cdot 10^{-9}$ as method parameters, and we perform a patch dynamics simulation using a macroscopic mesh size $\Delta x=2 \cdot 10^{-2}$ and $\Delta t=5 \cdot 10^{-3}$ using the upwind initialization (36), combined with forward Euler in time.

The simulations show that the patch dynamics scheme is a good approximation to a finite difference approximation of equation (16) in nonconservative form. In particular, the correct comparison scheme would be

$$
U_{i}^{n+1}=U_{i}^{n}-\Delta t\left(c^{*}\left(x_{i}\right) \frac{U_{i}^{n}-U_{i-1}^{n}}{\Delta x}+U_{i}^{n} \partial_{x} c^{*}\left(x_{i}\right)\right),
$$

which is not entirely the same as the classical finite volume upwind scheme

$$
U_{i}^{n+1}=U_{i}^{n}-\frac{\Delta t}{\Delta x}\left(c^{*}\left(x_{i+1 / 2}\right) U_{i}^{n}-c^{*}\left(x_{i-1 / 2}\right) U_{i-1}^{n}\right) .
$$

In particular, the scheme (44) is not conservative.

The results are shown in figure 9. Again, we note that the first-order upwind scheme is very diffusive, and that the error of the patch dynamics scheme with respect to the finite difference approximation (44) is 3 orders of magnitude smaller than the error with respect to the exact solution. Moreover, the error with respect to the finite difference scheme is an order of magnitude smaller than the error with respect to the finite volume scheme (45), which is consistent with the statements made above. 

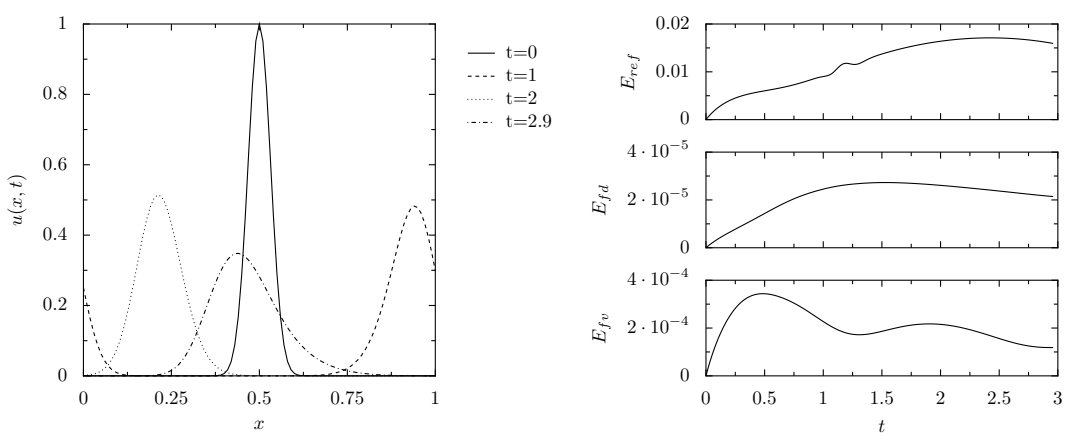

Fig. 9: Left: Snapshots of the solution of the homogenization advection equation (15) with coefficient (43) using the first-order upwind patch dynamics scheme at certain moments in time. Right: error with respect to the "exact" solution of the effective equation (16) (top), the finite difference comparison scheme (44) (middle) and the finite volume scheme (45) (bottom). The total error is dominated by the error of the finite difference scheme, and the error with respect to the finite volume scheme is significantly larger that the error with respect to (44).

\section{Conclusions}

In this paper, we reviewed the patch dynamics scheme and showed its basic convergence properties on model hyperbolic and parabolic homogenization problems. We illustrated that the scheme is capable of reproducing the correct macroscopic behaviour, even when the macroscopic equation is not of diffusion-type, and demonstrated that the required buffer size depends severely on the properties of the effective equation. Specifically, in the case of a macroscopic transport equation, the buffers can be very small compared to the diffusion case.

We wish to stress the fact that patch dynamics is an approximation to a finite difference scheme of the macroscopic equation in non-conservative form, which is most apparent in the case of coefficients that vary on a macroscopic scale. However, we note that there is no guarantee that the patch dynamics scheme will be conservative, even if the corresponding finite difference scheme is, since the extra errors that are induced might (and will) destroy conservation in the numerical solutions. When numerical conservation is important (e.g. if the macroscopic solution would develop sharp fronts), we will therefore need to resort to a finite volume formulation of the patch dynamics scheme. This variant is currently under active investigation.

\section{References}

1. A. Abdulle and W. E. Finite difference heterogeneous multi-scale method for homogenization problems. Journal of Computational Physics, 191(1):18-39, 2003. 
2. S. Attinger. Generalized coarse-graining procedures for flow in porous media. Computational Geosciences, 7:253-273, 2003.

3. I. Babuska. Homogenization and its applications. In B. Hubbard, editor, SYNSPADE, pages 89-116. 1975.

4. A. Bensoussan, J.L. Lions, and G. Papanicolaou. Asymptotic analysis of periodic structures, volume 5 of Studies in Mathematics and its Applications. NorthHolland, Amsterdam, 1978.

5. D. Cioranescu and P. Donato. An introduction to homogenization. Oxford University Press, 1999.

6. M. Dorobantu and B. Engquist. Wavelet-based numerical homogenization. SIAM J. Numer. Anal., 35(2):540-559, 1998.

7. W. E and B. Engquist. The heterogeneous multi-scale methods. Comm. Math. Sci., 1(1):87-132, 2003.

8. B. Engquist. Computation of oscillatory solutions to hyperbolic differential equations. Springer Lecture Notes Math., 1270:10-22, 1987.

9. B. Engquist and O. Runborg. Wavelet-based numerical homogenization with applications. In Multiscale and Multiresolution Methods, volume 20 of Lecture Notes in Computational Science and Engineering, pages 97-148. Springer, Berlin, 2002.

10. R. Erban and H.G. Othmer. From individual to collective behavior in bacterial chemotaxis. SIAM Journal on Applied Mathematics, 65(2):361-391, 2004.

11. C.W. Gear, T.J. Kaper, I.G. Kevrekidis, and A. Zagaris. Projecting to a slow manifold: Singularly perturbed systems and legacy codes. SIAM Journal on Applied Dynamical Systems, 4(3), 2005.

12. C.W. Gear and I.G. Kevrekidis. Constraint-defined manifolds: a legacy code approach to low-dimensional computation. J. Sci. Comp., 2004. In press.

13. C.W. Gear, I.G. Kevrekidis, and C. Theodoropoulos. "Coarse" integration/bifurcation analysis via microscopic simulators: micro-Galerkin methods. Computers and Chemical Engineering, 26:941-963, 2002.

14. G.H. Golub and C.F. Van Loan. Matrix computations (3rd ed.). Johns Hopkins University Press, Baltimore, MD, USA, 1996.

15. A.C. Hindmarsh. ODEPACK, a systematized collection of ODE solvers. In R.S. Stepleman et al., editor, Scientific Computing, pages 55-64. North-Holland, Amsterdam, 1983.

16. T.Y. Hou and X.H. Wu. A multiscale finite element method for elliptic problems in composite materials and porous media. Journal of Computational Physics, 134:169-189, 1997.

17. T.Y. Hou and X.H. Wu. Convergence of a multiscale finite element method for elliptic problems with rapidly oscillating coefficients. Mathematics of Computation, 68(227):913-943, 1999.

18. W. Hundsdorfer and J.G. Verwer. Numerical solution of time-dependent advection-diffusion-reaction equations, volume 33 of Springer Series in Computational Mathematics. Springer, 2003.

19. B. Jourdain, T. Lelièvre, and C. Le Bris. Numerical analysis of micro-macro simulations of polymeric fluid flows: a simple case. Mathematical Models and Methods in Applied Sciences, 12(9):1205-1243, 2002.

20. R. Keunings. Micro-macro methods for the multiscale simulation of viscoelastic flows using molecular methods of kinetic theory. In D.M. Binding and K. Walters, editors, Rheology Reviews, pages 67-98. British Society of Rheology, 2004. 
21. I.G. Kevrekidis, C.W. Gear, J.M. Hyman, P.G. Kevrekidis, O. Runborg, and C. Theodoropoulos. Equation-free, coarse-grained multiscale computation: enabling microscopic simulators to perform system-level tasks. Comm. Math. Sciences, 1(4):715-762, 2003.

22. P. Van Leemput, K. Lust, and I.G. Kevrekidis. Coarse-grained numerical bifurcation analysis of lattice boltzmann models. Physica D, 210(1-2):58-76, 2005.

23. P. Van Leemput, W. Vanroose, and D. Roose. Initialization of a latticeBoltzmann model with constrained runs. Journal of Computational Physics, 2005. Submitted.

24. J. Li, P.G. Kevrekidis, C.W. Gear, and I.G. Kevrekidis. Deciding the nature of the "coarse equation" through microscopic simulations: the baby-bathwater scheme. SIAM Multiscale modeling and simulation, 1(3):391-407, 2003.

25. A.G. Makeev, D. Maroudas, A.Z. Panagiotopoulos, and I.G. Kevrekidis. Coarse bifurcation analysis of kinetic Monte Carlo simulations: a lattice-gas model with lateral interactions. J. Chem. Phys., 117(18):8229-8240, 2002.

26. A.M. Matache, I. Babuska, and C. Schwab. Generalized p-FEM in homogenization. Numerische Mathematik, 86(2):319-375, 2000.

27. N. Neuss, W. Jäger, and G. Wittum. Homogenization and multigrid. Computing, 66(1):1-26, 2001.

28. H.C. Öttinger. Stochastic processes in polymeric fluids. Springer, 1996.

29. A.J. Roberts and I.G. Kevrekidis. Higher order accuracy in the gap-tooth scheme for large-scale solutions using microscopic simulators. Can be obtained as math.DS/0410310 from arxiv.org., 2004.

30. O. Runborg, C. Theodoropoulos, and I.G. Kevrekidis. Effective bifurcation analysis: a time-stepper based approach. Nonlinearity, 15:491-511, 2002.

31. G. Samaey, I.G. Kevrekidis, and D. Roose. Damping factors for the gap-tooth scheme. In S. Attinger and P. Koumoutsakos, editors, Multiscale Modeling and Simulation, volume 36 of Lecture Notes in Computational Science and Engineering, pages 93-102. Springer, 2004.

32. G. Samaey, I.G. Kevrekidis, and D. Roose. Patch dynamics with buffers for homogenization problems. Journal of Computational Physics, 2005. In press.

33. G. Samaey, D. Roose, and I.G. Kevrekidis. The gap-tooth scheme for homogenization problems. SIAM Multiscale Modeling and Simulation, 4(1):278-306, 2005.

34. C. Schwab and A.M. Matache. Multiscale and Multiresolution methods, volume 20 of Lecture Notes in Computational Science and Engineering, chapter Generalized FEM for homogenization problems, pages 197-238. Springer-Verlag, 2002.

35. C.I. Siettos, M.D. Graham, and I.G. Kevrekidis. Coarse Brownian dynamics for nematic liquid crystals: bifurcation, projective integration and control via stochastic simulation. J. Chem. Phys., 118(22):10149-10157, 2003. can be obtained as cond-mat/0211455 at arxiv.org.

36. C. Theodoropoulos, Y.H. Qian, and I.G. Kevrekidis. Coarse stability and bifurcation analysis using time-steppers: a reaction-diffusion example. In Proc. Natl. Acad. Sci., volume 97, pages 9840-9845, 2000. 